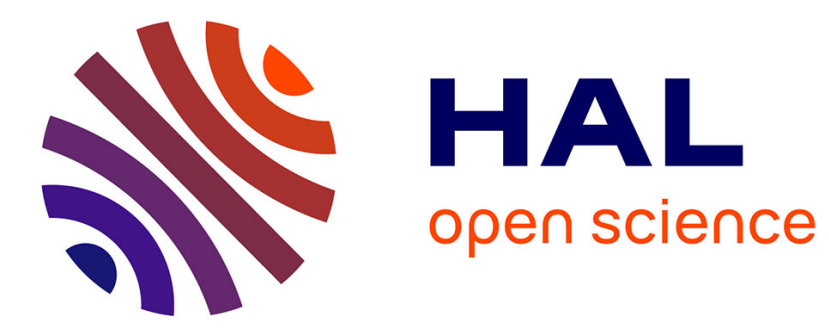

\title{
The excitation of NH2 in the interstellar medium
}

\author{
N Bouhafs, A Bacmann, A Faure, François Lique
}

\section{To cite this version:}

N Bouhafs, A Bacmann, A Faure, François Lique. The excitation of NH2 in the interstellar medium. Monthly Notices of the Royal Astronomical Society, 2019, 10.1093/mnras/stz2586 . hal-03064773

\section{HAL Id: hal-03064773 https://hal.science/hal-03064773}

Submitted on 14 Dec 2020

HAL is a multi-disciplinary open access archive for the deposit and dissemination of scientific research documents, whether they are published or not. The documents may come from teaching and research institutions in France or abroad, or from public or private research centers.
L'archive ouverte pluridisciplinaire HAL, est destinée au dépôt et à la diffusion de documents scientifiques de niveau recherche, publiés ou non, émanant des établissements d'enseignement et de recherche français ou étrangers, des laboratoires publics ou privés. 


\title{
The excitation of $\mathrm{NH}_{2}$ in the interstellar medium
}

\author{
N. Bouhafs ${ }^{1}$, A. Bacmann ${ }^{2}$, A. Faure ${ }^{2}$ and F. Lique ${ }^{2 \star}$ \\ ${ }^{1}$ LOMC - UMR 6294, CNRS-Université du Havre, 25 rue Philippe Lebon, BP 1123, 76063 Le Havre cedex, France \\ ${ }^{2}$ UJF-Grenoble 1/CNRS, Institut de Planétologie et d'Astrophysique de Grenoble (IPAG) UMR 5274, Grenoble F-38041, France
}

Accepted XXXX month XX. Received XXXX month XX; in original form XXXX month XX

\begin{abstract}
Accurate estimation of the abundance of the $\mathrm{NH}_{2}$ radical in the interstellar medium requires accurate radiative and collisional rate coefficients. The calculation of hyperfine-resolved rate coefficients for the collisional (de-)excitation of $\mathrm{NH}_{2}$ by both ortho- and para- $\mathrm{H}_{2}$ are presented in this work. Hyperfine-resolved rate coefficients are calculated from pure rotational close-coupling rate coefficients using the $M_{j}$ randomizing approximation. Rate coefficients for temperatures ranging from 5 to $150 \mathrm{~K}$ were computed for all hyperfine transitions among the first 15 rotational energy levels of both ortho- and para- $\mathrm{NH}_{2}$ in collisions with ortho- and para- $\mathrm{H}_{2}$. The new data were then employed in radiative transfer calculations to simulate the excitation of $\mathrm{NH}_{2}$ in typical star-forming regions such as W31C. We compared the excitation and brightness temperatures for different $\mathrm{NH}_{2}$ transitions obtained using the new and the previously available collisional data. It is found that the new rate coefficients increase the simulated line intensities. As a consequence, $\mathrm{NH}_{2}$ abundance derived from the observations will be significantly reduced by the use of the present rate coefficients.
\end{abstract}

Key words: Molecular data - Molecular processes - radiative transfer - scattering.

\section{INTRODUCTION}

Observations of nitrogen hydrides are crucial to constrain the nitrogen chemistry in space since they are at the root of the nitrogen chemical network. Among nitrogen hydrides, $\mathrm{NH}_{2}$ radical is one of the key molecules to understand such chemistry. Indeed, $\mathrm{NH}_{2}$ is a light and floppy molecule thought to be an important reactant intermediate in the production/destruction of nitrogen-bearing molecules such as ammonia $\left(\mathrm{NH}_{3}\right)$.

Interstellar $\mathrm{NH}_{2}$ was first detected by van Dishoeck et al. (1993) in absorption towards Sgr B2. Further, absorption lines of $\mathrm{NH}_{2}$ were observed through the use of the long-wavelength spectrometer aboard ISO (Cernicharo et al. 2000; Goicoechea et al. 2004; Polehampton et al. 2007). More recently, the Herschel Space Observatory (Pilbratt et al. 2010) with the Heterodyne Instrument for the Far-Infrared (HIFI, De Graauw et al. 2010)) offered the possibility to observe the ground-state rotational transitions with resolved hyperfine structure of $\mathrm{NH}_{2} . \mathrm{NH}_{2}$ is also well known in comets (Magee-Sauer et al. 1989; Swings et al. 1943), and have been observed in stellar photospheres via it's electronic, vibrationrotation, and high rotational transitions.

Moreover, $\mathrm{NH}_{2}$ exhibit spin symmetry states whose relative abundances are sensitive to the $\mathrm{H}_{2}$ ortho-to-para ratio

* E-mail: francois.lique@univ-lehavre.fr
(OPR) in the gas phase. $\mathrm{NH}_{2}$ observations may then also be able to put constraints on the $\mathrm{H}_{2}$ OPR in dense gas.

The absorption lines detected were employed to derive $\mathrm{NH}: \mathrm{NH}_{2}: \mathrm{NH}_{3}$ abundance ratios of $\sim 2: 1: 1$ in lukewarm diffuse clouds (Persson et al. 2010) and $\sim 3: 1: 20$ in the cold envelope of low-mass protostars (Le Gal et al. 2014). Emission lines were also detected more recently in denser and hotter interstellar regions (Persson et al. 2016). Anomalous (i.e. non statistical) ortho-to-para ratios of $\mathrm{NH}_{2}$ and $\mathrm{NH}_{3}$ were derived in the diffuse gas (Persson et al. 2012; Persson et al. 2016). These ratios were however successfully reproduced by gas-phase models including a rigorous nuclear-spin chemistry (Faure et al. 2013; Le Gal et al. 2014), suggesting that $\mathrm{NH}_{2}$, just like $\mathrm{NH}_{3}$, is mainly formed in the gas-phase via a series of successive hydrogen abstraction $\mathrm{NH}_{n}^{+}+\mathrm{H}_{2}$ $(n=0-3)$ reactions followed by electronic dissociation recombination. A recent work has also emphasized the importance of the $\mathrm{H}$-exchange reaction $\mathrm{NH}_{2}+\mathrm{H}$ in the ortho-para conversion of $\mathrm{NH}_{2}$ (Le Gal et al. 2016).

Due to the low density of the interstellar medium (ISM) $\left(\simeq 10^{2}-10^{6} \mathrm{~cm} 3\right)$, the energy levels of the $\mathrm{NH}_{2}$ molecule are expected to be out local thermodynamical equilibrium (LTE). Hence, deriving $\mathrm{NH}_{2}$ abundance from absorption and emission spectral lines requires excitation calculations that include collisional rate coefficients for transitions between the molecular energy levels induced by the most abundant ISM species, such $\mathrm{H}_{2}$. As no collisional data were available 
for the $\mathrm{NH}_{2}$ molecule and in order to perform non-LTE analysis of the $\mathrm{NH}_{2}$ observations, Persson et al. (2016) crudely estimated rate coefficients assuming a quenching rate coefficient of $5 \times 10-11 \mathrm{~cm}^{3} \mathrm{~s}^{-1}$ and state-specific downward rate coefficients for radiatively allowed transitions that scale in proportion to radiative line strengths.

However, it has been shown that the degree of excitation of molecules depends crucially on the set of collisional rate coefficients used (Roueff \& Lique 2013). Consequently, inaccurate rates may induce important errors in the determination of the molecular abundance in molecular clouds. Hence, considering the sensitivity of the radiative transfer models to the collisional rate coefficients, it is very important to provide accurate values for the $\mathrm{NH}_{2}$ collisional rate coefficients.

Recently, we presented quantum close-coupling calculations for the pure rotational excitation of $\mathrm{NH}_{2}$ due to collisions with $\mathrm{H}_{2}$ molecules (Bouhafs et al. 2017, hereafter Paper I). The calculations were based on a recent, high-accuracy full-dimensional $\mathrm{NH}_{4}$ potential energy surface (PES) adapted for rigid-rotor scattering calculations. Collisional cross sections were obtained for all transitions among the first 15 energy levels of both ortho- and para- $\mathrm{NH}_{2}$ and for total energies up to $1500 \mathrm{~cm}^{-1}$.

In this paper, we generate the first realistic set of rate coefficients of $\mathrm{NH}_{2}$ in collisions with $\mathrm{H}_{2}$ from the collisional cross sections of Paper I. Hyperfine-resolved rate coefficients are calculated from pure rotational close-coupling rate coefficients using the $M_{j}$ randomizing approximation for temperatures ranging from 5 to $150 \mathrm{~K}$.

The paper is organized as follows. In Section 2, we briefly present theoretical approach used to generate the collisional data. Section 3 presents rate coefficients for selected rotational and hyperfine-resolved transitions in $\mathrm{NH}_{2}$ induced by collisions with ortho- and para- $\mathrm{H}_{2}$. Finally, section 4 is devoted to an astrophysical applications and the conclusions of this work are drawn in Section 5.

\section{METHODS}

The scattering calculations are based on a five-dimensional (5D) PES determined from the nine-dimensional global PES of $\mathrm{NH}_{4}$ ( $\mathrm{Li} \&$ Guo 2014). Ab initio calculations for the $\mathrm{NH}_{2}-\mathrm{H}_{2}$ complex in its ground electronic state were carried out at the explicitly correlated coupled cluster with single, double, and perturbative triple excitations [UCCSD(T)F12a] (Knizia et al. 2009) level of theory with augmented correlation-consistent polarized valence- triple- $\zeta$ (aug-ccpVTZ) basis set (Dunning Jr 1989; Kendall et al. 1992). The collision partners were considered as rigid with geometries corresponding to the ground vibrational state. We refer the reader to Li \& Guo (2014) and Paper I for more details about the PES.

In order to solve the close-coupling equations for scattering, the PES was expanded over angular functions using the expression (Phillips et al. 1994) :

$$
V\left(R, \theta, \varphi, \theta^{\prime}, \varphi^{\prime}\right)=\sum v_{p_{1} q_{1} p_{2} p}(R) t_{p_{1} q_{1} p_{2} p}\left(\theta, \varphi, \theta^{\prime}, \varphi^{\prime}\right)
$$

where $t_{p_{1} q_{1} p_{2} p}\left(\theta, \varphi, \theta^{\prime}, \varphi^{\prime}\right)$ is formed from coupled spherical functions $Y_{l i, m i}(\alpha, \beta)$ which are associated with the rotational angular momenta of $\mathrm{NH}_{2}$ and $\mathrm{H}_{2}$.
The global minimum of the 5D PES corresponds to the most stable configuration of the $\mathrm{NH}_{2}-\mathrm{H}_{2}$ complex so that $\mathrm{H}_{2}$ is approaching the $\mathrm{N}$ atom of the $\mathrm{NH}_{2}$ molecule along the $C_{2 v}$ axis with a depth of $213 \mathrm{~cm}^{-1}$.

The rotational energy levels of $\mathrm{NH}_{2}$ are labelled by three numbers: the angular momentum $N_{1}$ and the pseudoquantum numbers $k_{a}$ and $k_{c}$ which correspond to the projection of $N_{1}$ along the axis of the least and greatest moments of inertia, respectively. Two forms (ortho- and para- $\mathrm{NH}_{2}$ ) caused by the different relative orientations of the hydrogen nuclear spins exist. The para states correspond to $k_{a}+k_{c}$ odd and the ortho states to $k_{a}+k_{c}$ even.

The calculations of pure rotational cross sections (i.e. neglecting the fine and hyperfine structure) that will be used in this paper to generate the rotational and hyperfine rate coefficients were performed with the MOLSCAT program (Hutson \& Green 1994) using the close-coupling approach (Green 1975) (Paper I). Calculations were carried out for total energies ranging from 32 to $1500 \mathrm{~cm}^{-1}$. Transitions among both the 15 lowest levels of ortho- $\mathrm{NH}_{2}$ and para- $\mathrm{NH}_{2}$ were considered. The convergence of the cross-sections with respect to the propagation parameters and with the $\mathrm{NH}_{2}$ and $\mathrm{H}_{2}$ rotational basis has been discussed in details in Paper I.

Here, from the calculated rotational cross-sections, one can obtain the corresponding thermal rate coefficients at temperature $\mathrm{T}$ by an average over the collision energy $\left(E_{c}\right)$ :

$$
\begin{aligned}
k_{\beta \rightarrow \beta^{\prime}}(T)= & \left(\frac{8}{\pi \mu k_{B}^{3} T^{3}}\right)^{\frac{1}{2}} \\
& \times \int_{0}^{\infty} \sigma_{\beta \rightarrow \beta^{\prime}} E_{c} e^{-\frac{E_{c}}{k_{B} T}} d E_{c}
\end{aligned}
$$

where $\sigma_{\beta \rightarrow \beta^{\prime}}$ is the collisional cross section. $\beta \equiv N_{1} \alpha, j_{2}$ and $\beta^{\prime} \equiv N_{1}^{\prime} \alpha^{\prime}, j_{2}^{\prime}$, unprimed and primed quantum numbers label initial and final states of the molecules, $j_{2}$ is the rotational angular momenta of $\mathrm{H}_{2}$, and $\alpha$ specifies the $\mathrm{NH}_{2}$ quantum numbers (e.g. $k_{a}, k_{c}$ ). $\mu$ is the reduced mass of the system and $k_{B}$ is the Boltzmann's constant. Calculations up to $1500 \mathrm{~cm}^{-1}$ allow determining rotation rate coefficients from 5 to $150 \mathrm{~K}$. The fine/hyperfine structure of the molecule was then considered using a statistical weight approximation that is presented below.

The electronic ground state of $\mathrm{NH}_{2}$ is ${ }^{2} B_{1}$ with a net unpaired electronic spin of $1 / 2$, Hence, each rotational level is split by spin-rotation interaction in a fine structure of two sublevels identified by the the total angular momentum $J_{1}$ with $J_{1}=N_{1} \pm S$ (where $N_{1}$ is the rotational angular momentum, and $S=1 / 2$ is the electronic spin). As the nitrogen atoms possess a non-zero nuclear spin $\left(I_{N}=1\right)$, the fine structure levels are further split into hyperfine levels, due to the coupling between the nitrogen nuclear spin and the total angular momentum $J_{1}$ characterized by the quantum number $F_{1}\left(F_{1}=J_{1} \pm I_{N}, I_{N}\right.$ being the nuclear spin of the first nitrogen atom). The ortho-transitions also have additional splitting due to the the coupling with the net proton spin identified by the quantum number $F\left(F=F_{1} \pm I_{H}\right.$, $I_{H}=I_{H_{1}}+I_{H_{2}}$ being the nuclear spin of the hydrogen atom).

Since $\mathrm{NH}_{2}$ hyperfine resolved rate coefficients can be crucial in detailed models of radiative transfer and in order to obtain a first overview of the impact of collisional rates on the astrophysical models, we have estimated from 
the rotational collision rates the hyperfine collision rates using the so-called $\mathrm{Mj}$ randomizing limit. It is a statistical weight approximation which assumes that the hyperfine de-excitation rate coefficients are proportional to the degeneracy of the final hyperfine level (here, $2 F^{\prime}+1$ ) and completely independent of the initial hyperfine level. Then, the de-excitation rate coefficients between hyperfine structure levels $\left(K_{N_{1 k a k c J_{1} F_{1} F}^{R A N} \rightarrow N_{1 k a^{\prime} k c^{\prime} J_{1}^{\prime} F_{1}^{\prime} F^{\prime}}^{\prime}}\right)$ are related to the close-coupling rate coefficients between rotational structure levels as follows (Faure \& Lique 2012):

$$
\begin{aligned}
& K_{N_{1 k a k c J_{1} F_{1} F}^{R A N} \rightarrow N_{1 k a^{\prime} k c^{\prime} J_{1}^{\prime} F_{1}^{\prime} F^{\prime}}^{\prime}}^{R A}= \\
& \quad \frac{\left(2 F^{\prime}+1\right)}{\left(2 I_{H}+1\right)\left(2 I_{N}+1\right)(2 S+1)\left(2 N_{1}^{\prime}+1\right)} K_{N_{1 k a k c} \rightarrow N_{1 k a}^{\prime}}^{C C}
\end{aligned}
$$

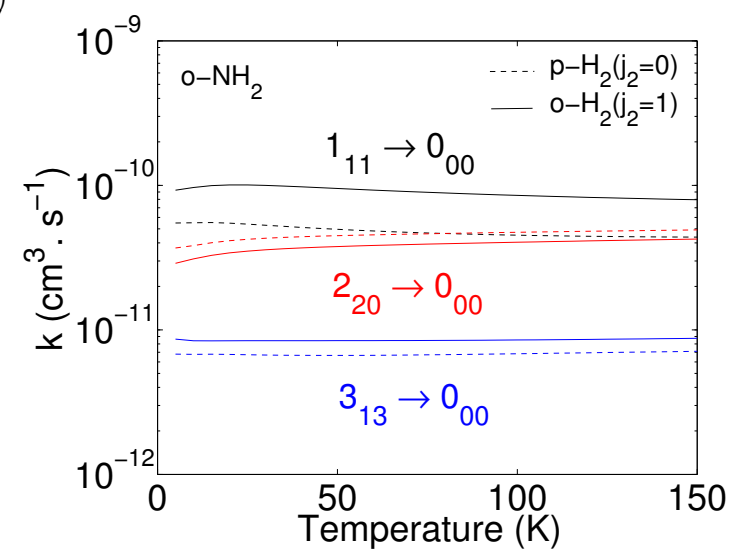

Figure 1. Typical rotational de-excitation rate coefficients of oand $\mathrm{p}-\mathrm{NH}_{2}$ by $\mathrm{H}_{2}\left(j_{2}=0,1\right)$ as a function of temperature

and 88 first hyperfine levels for para- $\mathrm{NH}_{2}$. The hyperfinestructure resolved rate coefficients display the same behavior than the rotationally inelastic rate coefficients. In particular, there is no temperature effect and the rate coefficients for collisions with para- $\mathrm{H}_{2}$ and ortho- $\mathrm{H}_{2}$ differ significantly, wich was expected since the hyperfine de-excitation rate coefficients are proportional to the degeneracy of the final hyperfine state.

Therefore, the present results can be considered as a reasonable first estimate of collisional hyperfine rate coefficients, since the statistical method provide hyperfine resolved rate coefficients with an average accuracy, with respect to the memory and CPU cost of full recoupling calculations, and represents a suitable and low-cost alternative for this system. On the other hand, in terms of radiative transfer application, it should be noted that at moderate and high opacities, where the relative hyperfine populations can significantly depart from the statistical weights, the SIOS method is notably better than the statistical approach (Faure \& Lique 2012).

The complete set of (de-)excitation rate coefficients will be available online from the LAMDA (Schöier et al. 2005) and BASECOL (Dubernet et al. 2013) websites. 


\section{THE EXCITATION OF $\mathrm{NH}_{2}$}

As a first simple application, we have carried out radiative transfer calculations to simulate the excitation of $\mathrm{NH}_{2}$ in interstellar molecular clouds. In particular, we focus on the physical conditions encountered in the massive star-forming region $\mathrm{W} 31 \mathrm{C}$ that is a complex and extremely luminous submillimetre and infrared continuum source. The more highly excited ortho-lines are detected in emission from the hot cores, while the lowest ortho- and para- $\mathrm{NH}_{2}$ lines at 953 and $1444 \mathrm{GHz}$ are in absorption and arise from the envelopes surrounding the hot cores.

Non-LTE calculations were performed with the RADEX code (Van der Tak et al. 2007) using the large velocity gradient (LVG) that simplifies the resolution of the statistical equilibrium equations by assuming that the velocity gradient is large enough to decouple the radiative transfer and the rotational level populations through the introduction of a photon escape probability.

Both collisional and radiative processes are taken into account. Einstein coefficients and frequencies were obtained from the Cologne Data base for Molecular Spectroscopy (Müller et al. 2005).

We include the cosmic microwave background radiation at $2.73 \mathrm{~K}$ and we assume a uniform spherical geometry of the cloud. A typical line width of $8.0 \mathrm{~km} \mathrm{~s}^{-1}$ was considered.

We have chosen different kinetic temperatures (30 and $100 \mathrm{~K}$ ) for the radiative transfer calculations. We varied the column density of otho- and para- $\mathrm{NH}_{2}$ from $10^{12}$ to $10^{14}$ $\mathrm{cm}^{-2}$ and we consider molecular hydrogen densities in a range from $10^{2}$ to $10^{12} \mathrm{~cm}^{-3}$.

In the radiative transfer calculations, we include all the energy levels for which both radiative and collisional data are available.

Figs. 2 displays the excitation temperatures $\left(\mathrm{T}_{e x}\right)$ and brightness temperatures $\left(\mathrm{T}_{B}\right)$ of the observed transition $N_{1 k a k c} J_{1}=1_{11} 3 / 2$ as a function of molecular hydrogen density for temperatures of 30 and $100 \mathrm{~K}$.

These hyperfine lines display classic behavior as a function of density. The excitation temperatures tend to be equal to the adopted value of background radiation field $(2.73 \mathrm{~K})$ at very low volume densities of molecular hydrogen. The excitation temperatures increase to higher values with increasing $\mathrm{H}_{2}$ density, and hence the rate of collisional excitation. At densities greater than a critical value, where the rates of photon and collisional excitation become equal, the excitation temperatures approach the kinetic temperature, and then the LTE approximation becomes valid.

We have also found that the column density has almost no impact on the magnitude of the excitation temperature. In fact, the three curves cannot be distinguished in the plots. This can be explained by the fact that $\mathrm{NH}_{2}$ lines are optically thin for the physical conditions considered in this work. At low temperatures $(\sim 30 \mathrm{~K})$, the critical density lies at $\approx$ $10^{8}-10^{9} \mathrm{~cm}^{-3}$, while for higher temperatures, the critical density is slightly larger (up to a factor of 2).

Since the rate coefficients do not vary strongly with temperature, the critical densities are relatively independent of the temperature. These critical densities are considerably greater than typical densities in the interstellar medium. Hence, we expect that the $\mathrm{NH}_{2}$ lines are not thermalized, and hence a non-LTE analysis is required even for analysis
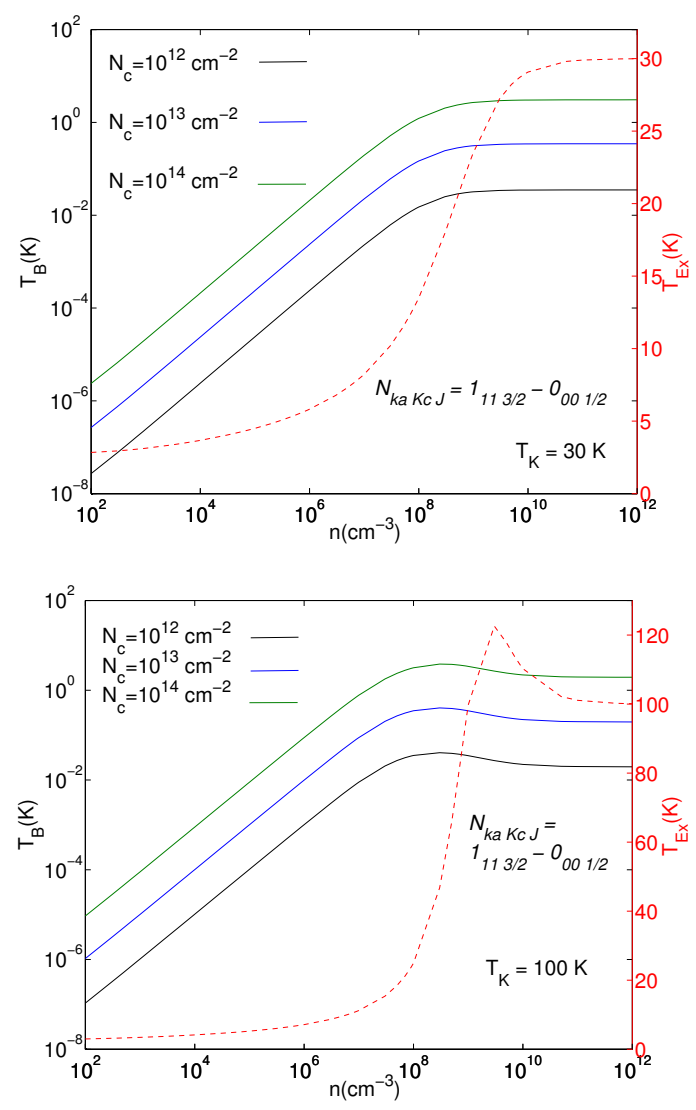

Figure 2. Excitation temperature $\left(\mathrm{T}_{E x}\right.$, dashed lines), brightness temperature $\left(\mathrm{T}_{B}\right.$, solid lines) for the lines of $\mathrm{NH}_{2}$. The $\mathrm{H}_{2}$ volume density varies between $10^{2}$ and $10^{12} \mathrm{~cm}^{-3}$ and the $\mathrm{NH}_{2}$ column density from $10^{12}$ to $10^{14} \mathrm{~cm}^{-2}$ by a step factor of 10 . The linewidth is $8 \mathrm{~km} \mathrm{~s}^{-1}$.

of absorption spectra, as it was shown in the previous analysis of NH absorption lines in the pre-stellar core $16293 \mathrm{~N}$ in L1689N (Bacmann et al. 2016). Figs 2 also show the brightness temperatures of the ortho- $\mathrm{NH}_{2}$ lines as a function of molecular hydrogen density. $\mathrm{T}_{B}$ increases gradually with the density before being constant at around $n\left(\mathrm{H}_{2}\right)=10^{7}-10^{8}$ $\mathrm{cm}^{-3}$ when LTE conditions are reached. Effect of suprathermal excitation is also seen at $\mathrm{T}_{k i n}=100 \mathrm{~K}$. This effect is due to the selection rules of the radiative and collisional transitions that will overpopulate one level with respect to another lower level and thus cause a sudden increase of the excitation temperature.

The two nuclear spin symmetries of $\mathrm{NH}_{2}$ have been observed towards the high-mass star-forming regions W31C using the Herschel-HIFI instrument (Persson et al. 2016). They detected three transitions with fine and hyperfine structure resolved of ortho- $\mathrm{NH}_{2}\left(N_{1 k a k c} J_{1}: 2_{11} 3 / 2 \rightarrow\right.$ $2_{02} 3 / 2,2_{02} 5 / 2 \rightarrow 1_{11} 3 / 2,4_{22} 9 / 2 \rightarrow 4_{13} 9 / 2,1_{11} 3 / 2 \rightarrow$ $\left.0_{00} 1 / 2\right)$ and one of para- $\mathrm{NH}_{2}\left(2_{12} 5 / 2 \rightarrow 1_{01} 3 / 2\right)$.

Persson et al. (2016) modeled the emission line profiles using collision rates of $\mathrm{NH}_{2}-\mathrm{H}_{2}$ estimated from an assumed quenching rate coefficient of $5 \times 10^{-11} \mathrm{~cm}^{3} \mathrm{~s}^{-1}$ and statespecific downward rates for radiatively allowed transitions that scale in proportion to radiative line strengths.

We were interested in the impact of the above approximate collisional rates on the excitation temperatures and 
peak intensity of $\mathrm{NH}_{2}$ hyperfine transitions, we have performed non-LTE radiative transfer calculations with the two sets of rate coefficients, namely the assumed data of Persson et al. (2016) and the present data.

From the observed spectra of W31C, we fix the basic parameters on which depend the LVG computation: the density of the collider $\mathrm{H}_{2}=10^{7} \mathrm{~cm}^{-3}$ (with an OPR =1), the kinetic temperature is kept fixed at $80 \mathrm{~K}$ (typical of hot core molecular clouds) and the column density of o- $\mathrm{NH}_{2}=210^{14}$ $\mathrm{cm}^{-2}$. The line width being set to $8 \mathrm{~km} \mathrm{~s}^{-1}$.

Table 1 presents excitation temperatures $\left(\mathrm{T}_{e x}\right)$ of the hyperfine lines associated with different $\mathrm{NH}_{2}$ rotational lines, computed with the present set of $\mathrm{NH}_{2}-\mathrm{H}_{2}$ and the earlier $\mathrm{NH}_{2}-\mathrm{H}_{2}$ rate coefficients of Persson et al. (2016).

In all cases, the excitation temperatures are greater than that of the CMB. Thus, these lines should be seen in emission, in agreement with the observations (Persson et al. 2016). All the lines are found to be optically thin.

It can be observed that the excitation temperatures and peak intensity based on the present rates are increased up to a factor of 30 with respect to those based on the assumed rates of Persson et al. (2016). These results clearly reflect the differences between the corresponding rates and highlight the importance of using computed $\mathrm{NH}_{2}-\mathrm{H}_{2}$ rate coefficients.

We conclude that the present rates will significantly improve the accuracy of the $\mathrm{NH}_{2}$ abudances and $\mathrm{H}_{2}$ densities derived from the observations and changes significantly the conclusions about the physical conditions in molecular clouds.

\section{CONCLUSION}

We have reported in this paper rate coefficients for the rotational excitation of ortho-, para- $\mathrm{NH}_{2}$ by ortho- and para$\mathrm{H}_{2}$. The lowest 15 rotational levels of ortho-, para- $\mathrm{NH}_{2}$ were included and kinetic temperatures up to $150 \mathrm{~K}$ were considered. Hyperfine resolved rate coefficients were also deduced using the $\mathrm{M}_{j}$ randomizing approximation. These calculations employed the PES for the interaction of $\mathrm{NH}_{2}$ with $\mathrm{H}_{2}$ computed by Li \& Guo (2014) using the [UCCSD(T)-F12a] coupled-cluster method.

In order to evaluate the effect of these new data on the astrophysical modelling, we have performed radiative transfer calculations corresponding to typical physical conditions of the ISM where $\mathrm{NH}_{2}$ emissions are detected. The simulated brightness temperatures obtained with our new rate coefficients are significantly larger than the ones obtained with previous rate coefficients assumed by Persson et al. (2016). The new rates lead to an increase by a factor of 1030 of the brightness temperatures, the $\mathrm{NH}_{2}$ abundance derived from the observations will be substantially smaller than the current estimates.

It would be useful to re-analyze the astronomical observations of $\mathrm{NH}_{2}$ rotational intensities to deduce the physical conditions in interstellar molecular clouds. A more complete treatment is now possible due to the availability of accurate $\mathrm{NH}_{2}-\mathrm{H}_{2}$ rate coefficients to model all detected lines, in absorption and emission, in order to improve the determination of the abundances and the physical conditions.

\section{REFERENCES}

Bacmann A., et al., 2016, Astronomy \& Astrophysics, 587, A26

Bouhafs N., Lique F., Faure A., Bacmann A., Li J., Guo H., 2017, The Journal of Chemical Physics, 146, 064309

Cernicharo J., Goicoechea J. R., Caux E., 2000, The Astrophysical Journal Letters, 534, L199

Cernicharo J., et al., 2011, Astronomy \& Astrophysics, 531, A103

De Graauw T., et al., 2010, Astronomy \& Astrophysics, 518, L6

Dubernet M.-L., et al., 2006, Astronomy \& Astrophysics, 460, 323

Dubernet M.-L., et al., 2013, Astronomy \& Astrophysics, 553, A50

Dunning Jr T. H., 1989, The Journal of chemical physics, 90, 1007

Faure A., Lique F., 2012, Monthly Notices of the Royal Astronomical Society, 425, 740

Faure A., Hily-Blant P., Le Gal R., Rist C., Des Forêts G. P., 2013, The Astrophysical Journal Letters, 770, L2

Goicoechea J. R., Rodríguez-Fernández N. J., Cernicharo J., 2004, The Astrophysical Journal, 600, 214

Green S., 1975, J. Chem. Phys., 62, 2271

Hutson J. M., Green S., 1994

Kendall R. A., Dunning Jr T. H., Harrison R. J., 1992, The Journal of chemical physics, 96, 6796

Knizia G., Adler T. B., Werner H.-J., 2009, The Journal of Chemical Physics, 130, 054104

Lanza M., Kalugina Y., Wiesenfeld L., Lique F., 2014, The Journal of chemical physics, 140, 064316

Le Gal R., Hily-Blant P., Faure A., des Forêts G. P., Rist C., Maret S., 2014, Astronomy \& Astrophysics, 562, A83

Le Gal R., Herbst E., Xie C., Li A., Guo H., 2016, Astronomy \& Astrophysics, 596, A35

Li J., Guo H., 2014, Physical Chemistry Chemical Physics, 16, 6753

Magee-Sauer K., Scherb F., Roesler F., Harlander J., Lutz B. L., 1989, Icarus, 82, 50

Müller H. S., Schlöder F., Stutzki J., Winnewisser G., 2005, Journal of Molecular Structure, 742, 215

Persson C. M., Black J. ., Cernicharo J., others. 2010, Astronomy and Astrophysics, 521, L45

Persson C. M., et al., 2012, Astronomy \& Astrophysics, 543, A145

Persson C. M., et al., 2016, A\&A, 586, A128

Phillips T. R., Maluendes S., McLean A., Green S., 1994, The Journal of chemical physics, 101, 5824

Pilbratt G., et al., 2010, Astronomy \& Astrophysics, 518, L1

Polehampton E. T., Baluteau J.-P., Swinyard B. M., Goicoechea J. R., Brown J. M., White G. J., Cernicharo J., Grundy T. W., 2007, Monthly Notices of the Royal Astronomical Society, 377, 1122

Roueff E., Lique F., 2013, Chemical reviews, 113, 8906

Schöier F. L., van der Tak F. F., van Dishoeck E. F., Black J. H., 2005, Astronomy \& Astrophysics, 432, 369

Swings P., McKellar A., Minkowski R., 1943, Astrophysical Journal, 98,142

Van der Tak F., Black J. H., Schöier F., Jansen D., van Dishoeck E. F., 2007, Astronomy \& Astrophysics, 468, 627

van Dishoeck E. F., Jansen D. J., Schilke P., Phillips T., et al., 1993

This paper has been typeset from a $\mathrm{T}_{\mathrm{E}} \mathrm{X} / \mathrm{LATEX}$ file prepared by the author. 
W31C

\begin{tabular}{|c|c|c|c|c|c|}
\hline \multirow[b]{2}{*}{$N_{k a k c} J_{1} F_{1} F \longrightarrow N_{k a^{\prime} k c^{\prime}}^{\prime} J_{1}^{\prime} F_{1}^{\prime} F^{\prime}$} & \multicolumn{2}{|c|}{ present work } & \multicolumn{2}{|c|}{ Persson et al.(2016) } & \multirow[b]{2}{*}{$T_{b_{1}} / T_{b_{2}}$} \\
\hline & $T_{e x}(\mathrm{~K})$ & $T_{b_{1}}(\mathrm{~K})$ & $T_{e x}(\mathrm{~K})$ & $T_{b_{2}}(\mathrm{~K})$ & \\
\hline $2_{11} 3 / 25 / 27 / 2 \longrightarrow 2_{02} 3 / 25 / 27 / 2$ & 26.60 & $1.32 \mathrm{E}-01$ & 13.13 & $3.99 \mathrm{E}-03$ & 33.07 \\
\hline $2113 / 25 / 25 / 2 \longrightarrow 2023 / 25 / 25 / 2$ & 27.40 & $5.53 \mathrm{E}-02$ & 12.94 & $1.83 \mathrm{E}-03$ & 30.30 \\
\hline $2_{11} 3 / 25 / 25 / 2 \longrightarrow 2_{02} 3 / 25 / 25 / 2$ & 31.70 & $2.64 \mathrm{E}-02$ & 12.80 & $8.73 \mathrm{E}-04$ & 30.25 \\
\hline $2113 / 23 / 25 / 2 \longrightarrow 2_{02} 3 / 23 / 25 / 2$ & 28.80 & $7.30 \mathrm{E}-02$ & 13.35 & $2.43 \mathrm{E}-03$ & 30.02 \\
\hline $2_{11} 3 / 21 / 23 / 2 \longrightarrow 2_{02} 3 / 21 / 23 / 2$ & 32.90 & $5.18 \mathrm{E}-02$ & 13.15 & $2.09 \mathrm{E}-03$ & 24.75 \\
\hline $2_{02} 5 / 27 / 29 / 2 \longrightarrow 1_{11} 3 / 25 / 27 / 2$ & 29.90 & $4.46 \mathrm{E}-01$ & 15.40 & 4.19E-02 & 10.64 \\
\hline $2_{02} 5 / 25 / 27 / 2 \longrightarrow 1_{11} 5 / 23 / 25 / 2$ & 43.50 & $3.10 \mathrm{E}-01$ & 15.30 & $3.40 \mathrm{E}-02$ & 9.12 \\
\hline $2_{02} 5 / 23 / 25 / 2 \longrightarrow 1_{11} 3 / 21 / 23 / 2$ & 47.40 & $2.33 \mathrm{E}-01$ & 14.97 & 3.53E-02 & 6.60 \\
\hline
\end{tabular}

Table 1. Comparison between excitation temperature and peak intensity for different ortho- $\mathrm{NH}_{2}$ observed transitions obtained with the assumed collisional rates of Persson et al. (2016) and those computed in this work. 